\title{
Care of Patients with Cystic Fibrosis
}

\section{Treatment, Screening and Clinical Outcome}

\author{
Birgitta Strandvik
}

Department of Pediatrics, Institute of Clinical Sciences, Sahlgrenska Academy, Göteborg University, Göteborg, Sweden

\section{Key Words}

Nutrition - Essential fatty acids • Staphylococcus aureus •

Pseudomonas aeruginosa $\cdot$ Intravenous treatment, at home •

Transplantation • Neonatal screening · Survival rate

\begin{abstract}
Cystic fibrosis (CF), the most common autosomal hereditary pediatric disease, is no longer restricted to childhood as modern therapy has given patients both increased longevity and better quality of life. When the gene was identified more than 16 years ago and the most common mutation, $\Delta \mathrm{F} 508$, found, general hope of a rapid cure for the disease was expressed. The discovery entailed a marked increase in research on pathophysiology, but to date the improvements in clinical therapy are entirely due to developments in traditional treatment. Improvements in the patient's condition are related to better pancreatic enzyme supplements and more intense nutritional treatment, including the attention paid to abnormal lipid metabolism. Pulmonary treatment has resulted in better preserved lung function into adulthood with access to a battery of efficient antibiotics and the development of new strategies; mucus-dissolving agents in combination with encouragement of physical activity from an early age together with modifications in physiotherapy, and improved results in lung transplantations as the ultimate treatment. Liver disease is recognized more, and often
\end{abstract}

treated long-term with ursodeoxycholic acid, although its effect in preventing progression of liver disease has not been fully explored. Neonatal screening is increasing, challenging short-term problems such as those of a psychological nature when carriers or very mild diseases are discovered, and exploring long-term results to prove that clinical status improves and survival further increases. The limitations in survival still present seem mainly to be due to the mystery behind the chronic colonization of the airways by microorganisms.

Copyright @ 2006 Nestec Ltd., Vevey/S. Karger AG, Basel

\section{Introduction}

When cystic fibrosis (CF) was identified as a separate disease from celiac disease in 1936-1938 [1, 2], treatment was very restricted and, consequently, mortality remained high during the first years of life [3]. Although the first patient to be diagnosed in adulthood was described in 1960 [4], there is still a widespread lack of knowledge about this disease among physicians, so mild cases might escape recognition, possibly throughout life. A problem in the treatment of CF patients is that very mild cases can also rapidly deteriorate after viral infections or due to other hitherto unrecognized factors, implying that once the diagnosis has been made, the pre-

Prof. Birgitta Strandvik

Department of Pediatrics, Queen Silvia Children's Hospital

SE-41685 Göteborg (Sweden)

Tel. +46 31343 4723, Fax +46 31217023

E-Mail karger@karger.ch Accessible online at:

www.karger.com www.karger.com/ane
E-Mail Birgitta.Strandvik@pediat.gu.se 
paredness to treat mild symptoms is important. This circumstance has ratified neonatal screening although still no cure is available. Because early pancreatic enzyme supplements showed unsatisfactory efficacy and nutritional problems were great, mortality in childhood was predominant and pediatric gastroenterologists had the chief responsibility in the care of CF patients. The development of pancreatic enzyme supplements in entericcoated microspheres resulted in improved efficacy, better absorption of nutrients, improved early survival, and thereby pulmonary symptoms received greater attention. During the 1970 s many new $\beta$-lactam antibiotics made it possible to focus more on pulmonary treatment and, with further development for treatment of the lung disease, prime care was increasingly taken over by pulmonologists. The improving results of lung transplantation will again turn the focus on gastrointestinal complications such as liver and biliary diseases, but also on other CFrelated problems, e.g. renal complications, bone fragility, diabetes and fertility problems. In turn this will elucidate the need to centralize the care of CF patients with a team of professionals, both within medical care and allied health professionals. As patients live longer and have better conditions for a normal social life, the treatment strategies have to be adapted to improve adherence to treatment. This confronts the professionals with new challenges in the care of CF patients.

\section{Organization of Care for Patients with CF}

Already in the 1960s, it was recognized that the centralized care of patients suffering from CF resulted in better survival, and close cooperation between CF centers and local doctors interested in CF problems can also contribute to improved care, provided that the patients are seen at least once a year at the center [5]. The reason is the nuances of the presenting symptoms, the recognition of which requires great experience of the practicing physician for optimal treatment. This also implies that a center for the care of CF patients should not be too small, i.e. not caring for less than 50 patients, and probably not too large either, since personal knowledge of the individual patient facilitates correct assessment of the actual status and the possible need for more intensive treatment. It has been shown that survival and the general condition of the patients are better if they are seen once a month for checkup compared to more infrequent visits. It is also well recognized today that a center should be organized as a team, consisting of a doctor, nurse, physiotherapist, dietician, social worker, psychologist, secretary, and technical assistant for sweat tests [6]. There is also a need for expert facilities, such as cooperation with geneticists, a bacteriological unit interested in the specific problems of culturing sputum from patients with CF, a clinical physiological unit interested in pulmonary assessments, a radiological unit, etc. A few CF centers should be closely affiliated with units performing lung and liver transplantations.

\section{Energy Requirements}

It is recommended that CF patients have an energy intake of $120 \%$ compared to healthy controls due to the increased basal metabolic rate [7]. The total energy expenditure differs depending on physical activity and impairment in lung function, but there are contradictory results regarding an association with genotype $[8,9]$. Some patients thrive without extra energy supplementation [10], although most studies show that in general CF patients have difficulties meeting even the recommended intake of energy, which might contribute to the difficulty in obtaining normal growth [11]. In this context it is worth noting that patients with mutations, such as $\Delta \mathrm{F} 508$, suggested as being associated with a high energy need, also have pancreatic insufficiency and seem to be more prone to develop essential fatty acid deficiency [12]. In rats, essential fatty acid deficiency is associated with increased energy expenditure.

To achieve a high energy intake, these patients need an energy-dense diet, i.e. a diet where fat contributes $40-$ $45 \%$ of the energy (E\%) as compared to at most $30 \mathrm{E} \%$ in healthy adults, but only a few patients have such a high fat intake [11]. However, the well-documented low plasma levels of linoleic acid (18:2n-6) need to be corrected. Therefore polyunsaturated fatty acids should contribute 10-15 E\%. A high intake of n- 6 fatty acids usually normalizes the low serum levels of docosahexaenoic acid [12, 13]. Patients with CF benefit from an increased intake of n-3 fatty acids, since the eicosanoids from eicosapentaenoic acid (20:5n-3), the prostaglandin $E_{3}$ and leukotriene- 5 series are anti-inflammatory and can counterbalance the increased eicosanoid production from the arachidonic acid (20:4n-6) cascade, which is increased in CF $[14,15]$, and most of which stimulate inflammation.

Increased physical activity, as a part of modern treatment, increases the need for energy. Pulmonary exacerbations with both an increased respiratory rate and the general impact of infection further increase the requirement for extra energy, more so in the severely ill patient. At the 
same time the increase in cytokines caused by infection may impair appetite, which often turns meals into a problem for CF patients [16]. This problem requires ingenuity in stimulating eating in order to prevent a shift to catabolic metabolism, which can rapidly become a condition demanding invasive measures, such as enteral nutrition.

The use of commercially available supplements as snacks can increase the energy intake but are not beneficial if they are used to reduce ordinary meals as their carbohydrate contents are comparably high and medium chain fatty acids (medium chain triglyceride fat) are no substitute for linoleic acid. In young children exacerbations of airway infections may temporarily impair the remaining pancreatic function and diarrhea or gastroenterological complaints can be an early symptom, indicating the need for more aggressive treatment of both the pancreatic insufficiency and the pulmonary infection.

Even with the pancreatic supplement therapy currently available, both in stable conditions and during exacerbations, it is quite often difficult to normalize fat absorption, indicating a need for further improvement in this therapy and also that other factors are involved in impaired fat absorption [17]. New pancreatic supplementations, from bacterial lipases and recombinant human bile salt-stimulated lipase, are being studied in clinical trials $[18,19]$. Severe liver impairment is rare, so a low bile acid synthesis as a significant contributing factor in impaired lipolysis is less likely [20]. However, biliary complications can decrease bile flow and therefore contribute to low intraluminal bile salt concentrations and malabsorption [21]. Another important factor might be that a low plasma linoleic acid concentration, indicating an essential fatty acid deficiency, might impair fat absorption by decreasing the transcellular transport of fat [22]. Due to defective absorption in essential fatty acid deficiency, i.e. low linoleic acid concentrations, it might even be necessary to supply linoleic acid intravenously during severe deficiency [23]. Patients with mutations related to severe phenotype are more prone to develop low plasma levels of linoleic acid and might more regularly need supplementation [12]. From a practical point of view, it might be recommended to use the intravenous access during antibiotic treatment of pulmonary exacerbations during the 3 last days of the course, and give $10 \mathrm{ml} \mathrm{10 \%} \mathrm{Intralipid} \mathrm{(or}$ the corresponding amount of comparable emulsions) $/ \mathrm{kg}$ body weight daily for $6-8 \mathrm{~h}$. It is important not to infuse too fast or during acute infections as the activity of the lipoprotein lipase may be reduced. In this context, special devices for infusion of emulsions are preferable. In patients with severe essential fatty acid deficiency, similar

CF Screening, Therapy and Clinical Outcome treatment can be recommended for 3 days every 2-3 weeks during 4-6 months for normalization of serum levels of linoleic acid with a concomitant improvement in renal and liver functions $[24,25]$.

In patients who have already developed malnutrition, usually in connection with severely impaired lung function, enteral nutrition (nasogastric tube or via gastroenterostomy) might be necessary to normalize anthropometry [26]. Energy-dense $(1 \mathrm{kcal} / \mathrm{ml})$ enteral nutrition formulas can preferably be given during the night so as not to interfere too much with ordinary food intake and ordinary life. Supplementation with enzymes before bedtime and the start of infusion is usually enough to give satisfying absorption. This is often a necessary treatment in patients waiting for transplantation, but might be even more efficient if given early for feeding problems, where the procedure can be temporary. Total parenteral feeding is usually not indicated.

\section{Vitamin Supplementation}

The difficulty of normalizing fat absorption necessitates extra supplementation of fat-soluble vitamins. Since CF patients have an increased oxidative capacity, the background of which is unknown [27], special attention has to be given to antioxidants, such as vitamin $\mathrm{E}$ and $\beta$ carotene, the concentrations of which are usually very low in CF. Vitamin A is important for mucosal integrity, but must be given special attention after transplantation when serum levels often become high. Serum concentrations of fat-soluble vitamins have to be monitored regularly, at least on a yearly basis, especially as there is concern that high levels might be toxic. For review see recent consensus reports summarizing nutritional practices $[28,29]$. Table 1 summarizes the recommendation for nutritional treatment.

\section{Handling Intestinal Complications}

Sometimes poor appetite is connected with recurrent abdominal pain, due to intestinal complications such as stenosis or distal intestinal obstruction syndrome. The former has to be treated surgically, but the latter can usually be successfully relieved by enema containing $10 \%$ $\mathrm{N}$-acetylcysteine and hyperosmolar radiological contrast solution in relation $1: 1$ or $2: 1 ; N$-acetylcysteine should not exceed $300 \mathrm{mg} / \mathrm{kg}$ body weight. In patients with frequent recurrences of symptoms, some help can be obtained from oral intake of large daily amounts of inert polymers, such as a mixture of different sizes of polyethylene glycol together with dietary adjustments avoiding fiber-rich food [30]. 
Table 1. Recommended daily nutrient intake in CF patients ${ }^{1}$

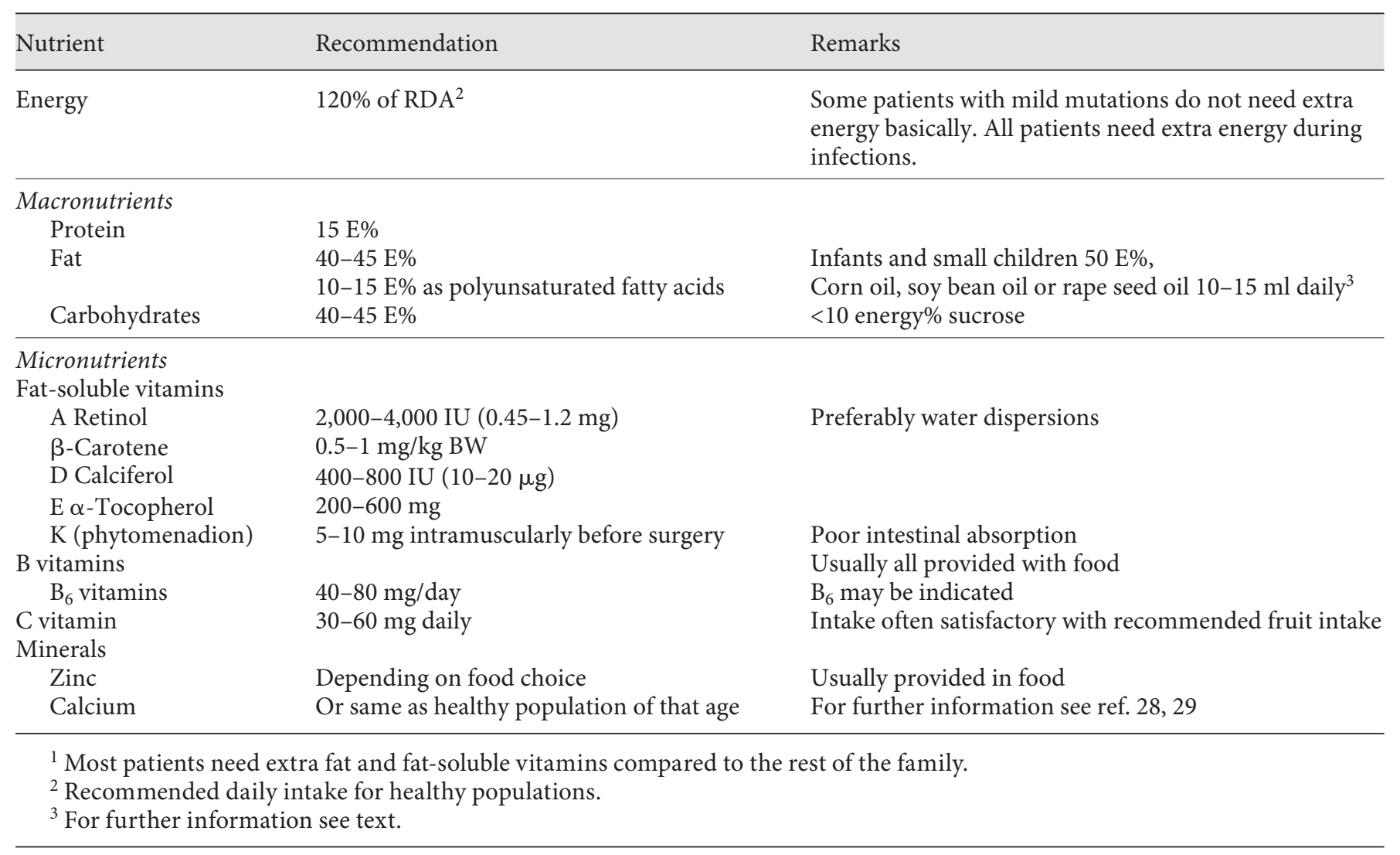

\section{Therapy of Lung Disease}

Treatment of airway symptoms has had most attention as pulmonary disease is responsible for $95 \%$ of the mortality in CF. Although all strategies rely on a combination of mucus-dissolving agents, passive or active physical activities and antibiotics, success varies greatly both between countries and centers [31]. Most attention has been paid to the chronic colonization with Pseudomonas aeruginosa, although the more or less chronic colonization with Staphylococcus aureus, which usually precedes the former, gives a much higher respiratory burst and therefore is also more tissue aggressive [32]. There is a general risk that the fear of Pseudomonas colonization, which demands intravenous antibiotic administration, causes a unconscious neglect to treat $S$. aureus. $P$. aeruginosa species are facultative aerobic bacteria, which are widespread in nature, and patients treated aggressively for mild symptoms can harbor them for decades without deterioration of pulmonary function [33]. Other common bac- teria in CF are Haemophilus influenzae and Stenotrophomonas maltophilia. The Burkholderia cepacia complex is a group of bacteria, some strains of which are connected with a severe and contagious course, often called the 'cepacia syndrome'.

The intensive long-term antibiotic treatments, developed at many centers, have resulted in problems with resistant strains. The fear of distribution of these strains between patients has resulted in the separation of patient units based on the bacterial colonization, causing great psychological stress to the patients $[34,35]$. A recent Cochrane report inquires whether an evaluation of the high antibiotic load, to keep $P$. aeruginosa intermittently in stead of chronically recognized, is justified in light of the problems with resistant strains and the segregation of patients [36]. A recent survey of treatment policy in the Nordic countries has shown that there are centers without this policy and they have a comparatively higher prevalence of Pseudomonas among their patients, but less of a problem with resistant strains [37]. These patients 
Table 2. Most commonly used pulmonary treatment strategies for mucolytics and antibiotics ${ }^{1}$

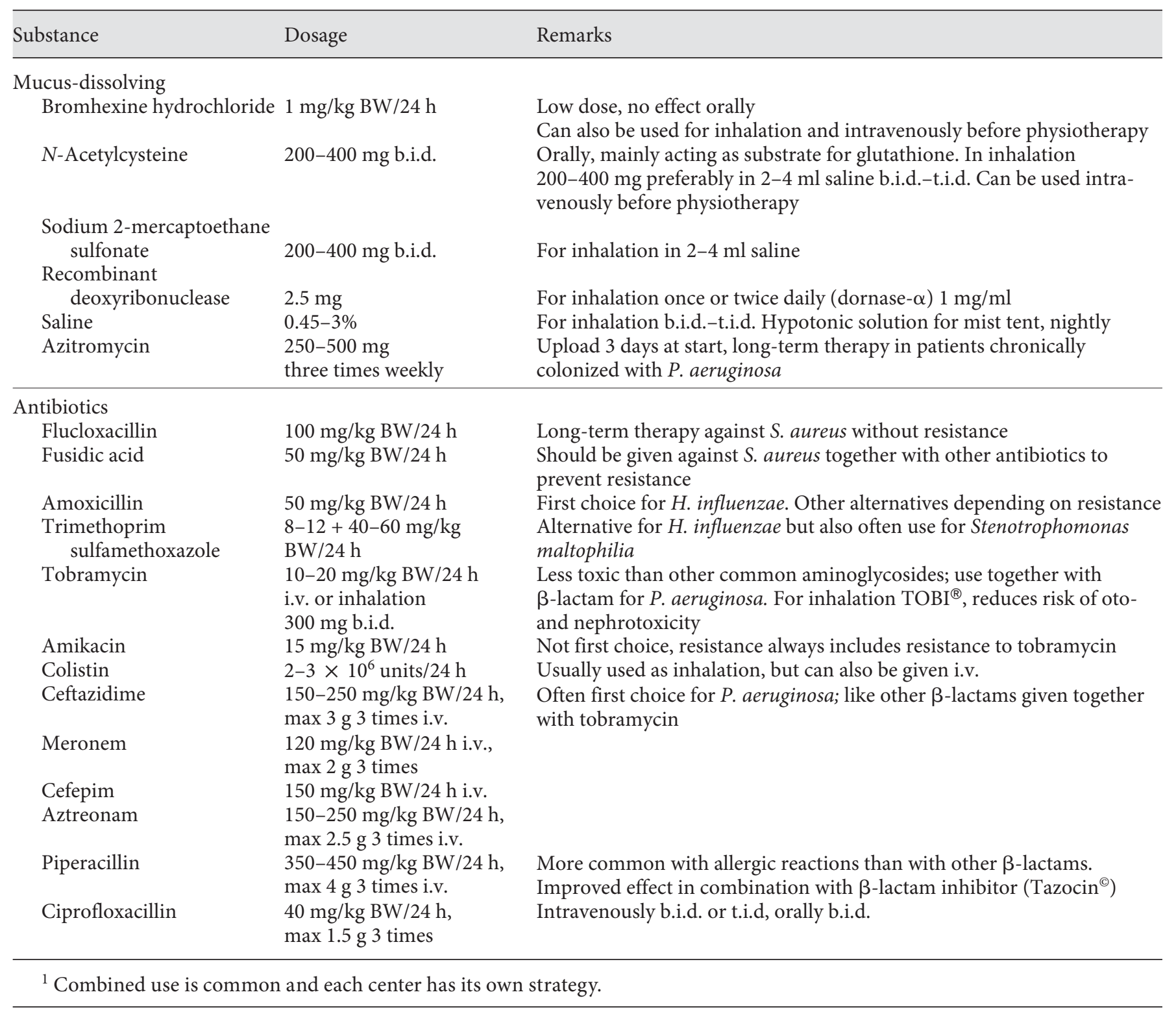

have good anthropometry and lung function and therefore also good quality of life, indicating that alternative treatment strategies might be worth considering [Lindblad A, et al., for the Scandinavian CF Consortium, to be published] (fig. 1).

Table 2 lists different agents commonly used in the treatment of the lung disease. Different treatment strategies have been developed, most focusing on preventing chronic colonization with gram-negatives, mainly $P$. aeruginosa. The strategies contain both generalized pulmonary mucus-dissolving agents, physical therapy and an- tibiotics. The very sticky mucus adheres to the mucosa and, as a substrate, contributes to bacterial colonization (fig. 2). At diagnosis, and to prevent this development, most centers start therapy with mucus-dissolving agents alone until isolation of bacteria is demonstrated. This treatment may involve physical activity [38], and may from an early age include physiotherapy on balls and trampolines. In some countries more passive physiotherapy with clapping using the daily help of physiotherapists is still dominant. From diagnosis on, the use of oral bromhexine prevents plugs caused by the sticky mucus accu- 


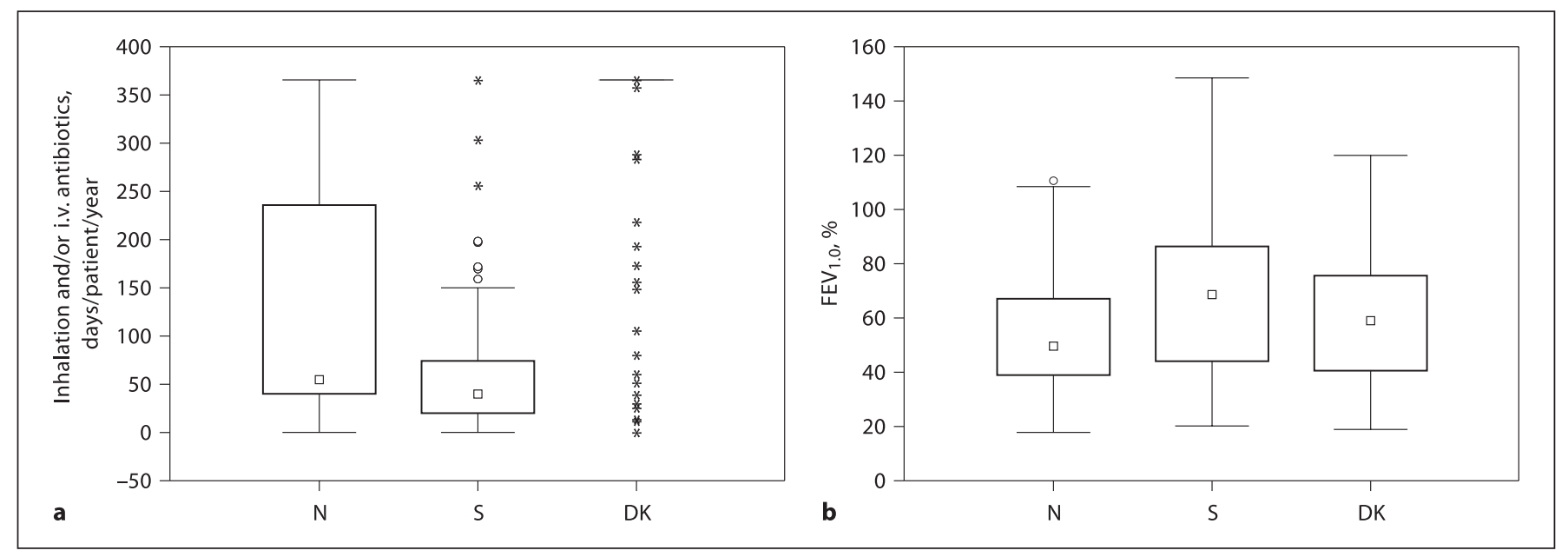

Fig. 1. Box plot of antipseudomonal therapy in patients aged $>19$ years and chronically colonized with $P$. aeruginosa in the three Nordic countries Norway (N), Sweden (S), and Denmark (DK; a) and the corresponding lung function expressed as forced expiratory volume in $1 \mathrm{~s}\left(\mathrm{FEV}_{1.0}\right)$ as a percentage of the predicted values (b). a The number of days with inhalation and/or intravenous antibiotics per patient and year. Medians and 25th and 75th percentiles are indicated. Whiskers indicate 5th and 95th percentiles; dots the outliers, and asterisks the extremes. Published with permission from the Scandinavian CF Consortium, Dr. A. Lindblad.

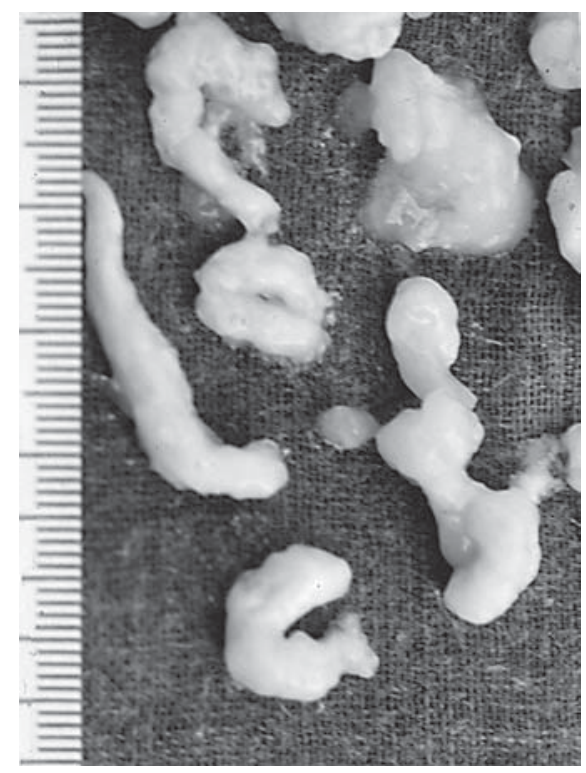

Fig. 2. Mucus plugs obtained by bronchoscopy from a child with cystic fibrosis. Photo: Dr. Jean Feigelson, Paris, with permission.

mulation and decreases symptoms of chronic sinusitis. Daily inhalation with saline, broncholytics and mucusdissolving agents is also introduced early; the frequency is usually determined by symptoms. All drugs have been shown to increase expectorations and improve lung function in patients with pulmonary symptoms, but their role as prophylaxis is less clear. From a clinical point of view, the choice of drug should be individualized, i.e. some patients have the best effect with dornase- $\alpha$ and others as good an effect with only saline $[39,40]$. For all inhalation therapy, a combination of physical activity and/or physiotherapy, also individualized for adherence, gives the best result for mucus expectoration. In recent years azithromycin has been used as a mucolytic. Its effect in patients chronically colonized with $P$. aeruginosa is remarkable, although the bacteria are not sensitive to the drug. Long-term administration has been able to reduce the number of intravenous antibiotic courses.

\section{Antibiotic Strategies}

Generally $S$. aureus is treated when isolated and the effect of treatment can easily be monitored by specific serum antibodies, such as those against teichoic acid and $\alpha$-toxin [41]. Since sputum samples might be difficult to obtain in small children, some centers give prophylactic long-term treatment during early childhood with fusidic acid or flucloxacillin, and some abstain because of fear that it might speed up colonization with gram-negative bacteria. It might be of importance to note that resistance to flucloxacillin is extremely rare, and countries like in Sweden which use this drug for long-term treatment still rarely isolate methicillin-resistant strains [37]. 
At first isolation of $P$. aeruginosa, aggressive treatment is a rule today, and chronic colonization can thereby be postponed. Most centers use intravenous antibiotics with a combination of aminoglycoside, usually tobramycin, and a $\beta$-lactam, followed by inhalation with tobramycin or colistin for weeks to months [42]. Some centers introduce regular tobramycin $\left(\mathrm{TOBI}^{\circledR}\right)$ inhalation in a 4-weeks-on and 4-weeks-off schedule. Others use a similar scheme for inhalation with colistin. The more intensive and long-term the treatment used, the less risk for chronic colonization (defined as more than one isolation of $P$. aeruginosa during 6 months and positive anti-pseudomonas antibody titers [43]), and instead intermittent colonization is achieved at the cost of high antibiotic consumption. This intensive antibiotic load also results in chronic colonization with other types of bacteria, such as Achromobacter xylosoxidans. As shown in figure 1 , in the Nordic country with the least antibiotic load, the patients' lung function was just as good as in the other countries, indicating that the strategy of how to use antibiotics is of great importance. In some severely ill patients with Pseudomonas strains of low susceptibility, continuous infusion of $\beta$-lactam can be used with good effects. Generally there is less risk of the development of resistance if the treatments are kept to 7-14 (mean 10) days, and when longer treatments are necessary the antibiotics should be changed $[33,37]$. In our experience there is less of a problem getting rid of mucoid strains than of non-mucoid strains. The reason is not obvious, but might be related to the strategy of using mucolytics and antibiotics.

Antibiotic treatment with intravenous antimicrobial drugs at signs of exacerbation of the pulmonary symptoms has been the most widely used treatment modality. In the struggle to keep the infectious load as low as possible, more or less continuous inhalation therapy has been more common in recent years. The development of a special preparation of tobramycin for inhalation therapy $\left(\mathrm{TOBI}^{\circledR}\right)$ has changed the treatment policy in many countries from intravenous administration to a longterm 4-weeks-on and 4-weeks-off schedule of antibiotic administration, and it has been shown to improve and keep pulmonary function rather constant for long periods of time [44]. Similar results can be obtained with traditional treatment using a combination of tobramycin and $\beta$-lactam, with less problems regarding antimicrobial resistance of the bacterial strains $[33,45]$. This is illustrated by the fact that the Nordic country with the lowest antibiotic consumption for CF patients rarely uses inhalation therapy. The costs of intravenous thera-

CF Screening, Therapy and Clinical Outcome py can also be kept relatively low by teaching parents and patients to perform home intravenous antibiotic therapy, which also gives the family more freedom and thereby greater adherence to and acceptance of treatment [46]. Also blood sampling, which is required during each course after the third dose of aminoglycoside has been administered to control for dose and make adjustments to avoid toxicity, can be taken at home and delivered to the hospital.

A problem sometimes arises when an allergic reaction to the preferred antimicrobial drug occurs, or even to the only one to which the bacteria show sensitivity in in vitro testing. This can be overcome by very careful desensitizing, starting with very low concentrations of the antibiotic during the actual course [47]. Longer treatment periods can be necessary, especially in severely ill patients awaiting pulmonary transplantation, and variation of antibiotics is then of importance.

\section{Steroids or Not}

Steroids are sometimes used to decrease the inflammation, but systemic administration increases the risk of clinical diabetes and should therefore be used with restriction. The use of new steroid formulations for inhalation has increased, but there are still no investigations showing if the anti-inflammatory effect of steroids is more effective than antibiotic treatment [48].

\section{Treatment of Hypoxemia}

Peripheral oximetry should be checked regularly as low oxygenation can appear insidiously, also when pulmonary function seems to be well preserved. The mechanisms behind this are not clear. The first sign usually develops at night during pulmonary exacerbations [49] and, although this is well recognized, there are no investigations in CF showing how this should be best treated [50]. Often patients with moderately impaired oxygenation are recommended nightly supplementation, but at some level continuous oxygen supply is necessary. While awaiting transplantation, pressure support ventilation treatment can be useful, and it can also be a necessary complement for severe exacerbations [51]. Assistant respiration with a machine is contraindicated as it may be impossible to extubate the patient.

\section{Indication for Lung Transplantation}

Patients should be considered for transplantation when their lung function is impaired to a $\mathrm{FEV}_{1.0}$ of $<30 \%$ of the predicted values. The decision to put the patient on the waiting list for lung transplantation must take many 
factors into account, such as nutrition, rate of decline in lung function, type of bacterial colonization, psychological factors, etc. [52].

\section{Screening}

Screening for CF was first introduced in the 1960s using determination of fecal albumin as an indicator of pancreatic insufficiency. It was never widely used because of major problems with both false-positive and false-negative results. An increase in immunoreactive trypsin in serum was found to have higher specificity and sensitivity, and it has recently been used as the first tool, sometimes repeated and usually followed up by mutation analysis of the most common regional presenting mutations before performance of the sweat test [53]. The purpose of introducing screening is to prevent the development of pulmonary damage, and short-term results have been positive both regarding nutrition and pulmonary disease [54], but after 10 years of age most studies show very little difference to unscreened patients [55]. This has been disappointing, and the most recent consensus report still does not generally recommend screening for general populations but finds it justified for regions and special purposes [53]. Many very careful follow-ups of screened populations have increased our knowledge about the natural history of the early disease manifestations. Problems following screening are: the diagnosis of cases without or with very mild early symptoms; the carrier diagnostics; early mother-infant bonding disturbances; inability to identify mild symptoms in patients missed by screening, and the costs [55]. As there still is no curative treatment for $\mathrm{CF}$, the introduction of screening has to be considered regionally, in close cooperation with CF centers, and balancing the costs towards the benefits for both the individual and society.

\section{Outcome}

CF is no longer a disease restricted to pediatrics. In an increasing number of centers, half or close to half of the patients are adults. The median survival rate has increased to at least the middle of the third decade both in the US and in Western Europe, and in some centers is even much higher [56]. However, there are great regional differences in the rest of the world, mostly due to socioeconomic factors; for instance in some countries of former Eastern Europe the absence of the most basic medi- cal treatment leaves no options for treatment. In the developed world most patients today have a good social life, manage to work full or part time, and also men with CF can raise a family due to successful sperm aspiration followed by in vitro fertilization. Although the incidence of CF-related diabetes increases with age, this type of diabetes is usually easy to treat and early recognition and treatment with short-reacting insulin can prevent the deterioration often reported with this complication. Liver disease virtually never influences survival of the patient. First of all it does not generally seem to progress with age and, if it does, the outcome of liver transplantation is now generally good, often also with improvement in lung symptoms [57]. Combined transplantations of lungs and liver are therefore rarely indicated, but recently combined transplantations of the pancreas and liver have been successful [58]. Longevity is further improved by the successful results of lung transplantation. CF patients generally have a better 5 -year survival than patients transplanted for other diseases, probably because they are younger and especially if they are also in good nutritional status $[59,60]$.

\section{Conclusion}

It is now more than 16 years since the gene defect behind this disease was discovered and, based on this a marked increase in knowledge has been gained from a remarkable increase in basic research. Several potential innovations have been made for more basic treatment of the disease, but to date the great improvement in survival is due to the continuous development of traditional treatment, centralized care, and the insight that frequent checkups of the patient can identify mild pulmonary symptoms. Aggressive treatment will prevent the development of progressive lung damage. In this context, special observation is necessary so that long-term treatment with inhalation of antibiotics and rarer visits to the clinic do not counteract the intention of the treatment. It is a difficult task for the center and team to motivate politicians to give resources to the right treatments and options for the very best interest of the patient. 


\section{References}

1 Fanconi G, Uehlinger E, Knauer C: Das Coeliakiesyndrom bei angeborenen zystischer Pancreasfibromatose und Bronchiektasien. Wien Med Wochenschr 1936;86:753-756.

2 Andersen DH: Cystic fibrosis of the pancreas and its relation to celiac disease: a clinical and pathological study. Am J Dis Child 1938; 56:344-399.

3 Bodian M: Fibrocystic Disease of the Pancreas: A Congenital Disorder of Mucus Production - Mucosis. New York, Grune \& Stratton, 1953.

$\checkmark 4$ Marks BL, Anderson CM: Fibrocystic disease of the pancreas in a man aged 46 . Lancet 1960;1:365.

$>5$ van Koolwijk LME, Uiterwaal CSPM, van der Laag J, et al: Treatment of children with cystic fibrosis: central, local or both? Acta Paediatr 2002;91:972-977.

$\checkmark 6$ Littlewood JM: Good care for people with cystic fibrosis. Paediatr Respir Rev 2000;1: 179-189.

$>7$ Thomson MA, Wilmott RW, Wainwright C, et al: Resting energy expenditure, pulmonary inflammation, and genotype in the early course of cystic fibrosis. J Pediatr 1996; 129:367-373

$>8$ O'Rawe A, McIntosh I, Dodge JA, et al: Increased energy expenditure in cystic fibrosis is associated with specific mutations. Clin Sci 1992;82:71-76.

$>9$ Fried MD, Durie PR, Tsui L-C, et al: The cystic fibrosis gene and resting energy expenditure. J Pediatr 1991;119:913-916.

10 Kindstedt-Arfwidson K, Strandvik B: Food intake in patients with cystic fibrosis on an ordinary diet. Scand J Gastroenterol 1988; 23:160-162

11 Kawchak DA, Zhao H, Scanlin TF, et al: Longitudinal, prospective analysis of dietary intake in children with cystic fibrosis. J Pediatr 1996;129:119-129.

12 Strandvik B, Gronowitz E, Enlund F, et al: Essential fatty acid deficiency in relation to genotype in patients with cystic fibrosis. J Pediatr 2001;139:650-655.

-13 Farrell PM, Mischler EH, Engle MJ, et al: Fatty acid abnormalities in cystic fibrosis. Pediatr Res 1985;19:104-109.

14 Kurlandsky LE, Bennink MR, Webb PM, et al: The absorption and effect of dietary supplementation with omega-3 fatty acids on serum leukotriene $\mathrm{B}_{4}$ in patients with cystic fibrosis. Pediatr Pulmonol 1994;18:211-217.

- 15 Strandvik B, Svensson E, Seyberth HW: Prostanoid biosynthesis in patients with cystic fibrosis. Prostagland Leukotr Essent Fatty Acids 1996;55:419-425.

-16 Stark LJ: Can nutrition counselling be more behavioural? Lessons learned from dietary management of cystic fibrosis. Proc Nutr Soc 2003;62:793-799.
17 Baker SS, Borowitz D, Duffy L, et al: Pancre atic enzyme therapy and clinical outcomes in patients with cystic fibrosis. J Pediatr 2005; 146:189-193.

18 Strandvik B, Hansson L, Hernell O, et al: Recombinant human bile salt-stimulated lipase improves lipid uptake and reduces the pancreatic enzyme supplementation in patients with cystic fibrosis. Pediatr Pulmonol 2004(suppl 27):333.

19 Borowitz D, Goss CH, Stevens C, et al: Safety and preliminary clinical activity of a novel pancreatic enzyme preparation in pancreatic insufficient cystic fibrosis patients. Pancreas 2006;32:258-263.

20 Strandvik B, Einarsson K, Lindblad A, Angelin B: Bile acid kinetics and biliary lipid composition in cystic fibrosis. J Hepatol 1996;25: 43-48.

21 Harries JT, Muller DPR, McCollum JPK, et al: Intestinal bile salts in cystic fibrosis. Arch Dis Child 1979;54:19-24.

22 Perin N, Jarocka-Cyrta E, Keelan M, et al: Dietary lipid composition modifies intestinal morphology and nutrient transport in young rats. J Pediatr Gastroenterol Nutr 1999;28:46-53.

23 Maqbool A, Schall J, Zemel B, et al: Essential fatty acid deficiency and the relevance of the triene:tetraene ratio in children with cystic fibrosis. J Pediatr Gastroenterol Nutr 2005; 41:550.

24 Strandvik B, Berg U, Kallner A, Kusoffsky E: Effect on renal function of essential fatty acid supplementation in cystic fibrosis. J Pediatr 1989;115:242-250.

25 Strandvik B, Hultcrantz R: Liver function and morphology during long term fatty acid supplementation in cystic fibrosis. Liver 1994;14:32-36.

26 van Biervliet S, De Waele K, van Winckel M, Robberecht E: Percutaneous endoscopic gastrostomy in cystic fibrosis: patient acceptance and effect of overnight tube feeding on nutritional status. Acta Gastroenterol Belg 2004;67:241-244.

27 Brown RK, Kelly FJ: Evidence for increased oxidative damage in patients with cystic fibrosis. Pediatr Res 1994;36:487-493.

28 Sinaasappel M, Stern M, Littlewood J, et al: Nutrition in patients with cystic fibrosis: a European Consensus. J Cyst Fibr 2002;1:5175.

29 Borowitz D, Baker RD, Stallings V: Consensus report on nutrition for pediatric patients with cystic fibrosis. J Pediatr Gastroenterol Nutr 2002;35:246-259.

30 Proesmans M, De Boeck K: Evaluation of dietary fiber intake in Belgian children with cystic fibrosis: Is there a link with gastrointestinal complaints? J Pediatr Gastroenterol Nutr 2002;35:610-614.

-31 Ferkol T, Rosenfeld M, Milla CE: Cystic fibrosis pulmonary exacerbations. J Pediatr 2006;148:259-264.
\$2 Hollsing AE, Lantz B, Bergström K, et al: Granulocyte elastase-alpha1-antiproteinase complex in cystic fibrosis: sensitive plasma assay for monitoring pulmonary infections. J Pediatr 1987;111:206-211.

33 Strandvik B: Antibiotic therapy of pulmonary infections in cystic fibrosis. Dosage and dose schedules. Chest 1988;94:146S-149S.

$\checkmark 34$ Koch C, Frederiksen B, Hoiby N: Patient cohorting and infection control. Semin Respir Crit Care Med 2003;24:703-716.

35 Steinkamp G, Ullrich G: Different opinions of physicians on the importance of measures to prevent acquisition of Pseudomonas aeruginosa from the environment. J Cyst Fibros 2003;2:199-205.

36 Wood DM, Smyth AR: Antibiotic strategies for eradicating Pseudomonas aeruginosa in people with cystic fibrosis. The Cochrane Database of Systematic Reviews 2006;DOI: 10.1002/14651858.CD004197.pub2.

37 Strandvik B, Hjelte L, Malmborg AS: Cystic fibrosis pulmonary infection: the Swedish experience; in Bauernfeind A, Marks MI, Strandvik B (eds): Cystic Fibrosis Pulmonary Infections: Lessons from Around the World. Basel, Birkhäuser, 1996, pp 293-302.

38 Blomquist M, Freyschuss U, Wiman LG, Strandvik B: Physical activity and self treatment in cystic fibrosis. Arch Dis Child 1986; 61:362-367.

39 Suri R: The use of human deoxyribonuclease (rhDNase) in the management of cystic fibrosis. BioDrugs 2005;19:135-144.

40 Elkins MR, Robinson M, Rose BR, et al: A controlled trial of long-term inhaled hypertonic saline in patients with cystic fibrosis. N Engl J Med 2006;354:229-240.

41 Hollsing AE, Granström M, Strandvik B: Prospective study of serum staphyloccocal antibodies in cystic fibrosis. Arch Dis Child 1987;62:905-911

42 Cantón R, Cobos N, de Gracia J, et al; on behalf of the Spanish Consensus Group for Antimicrobial Therapy in the Cystic Fibrosis Patient: Antimicrobial therapy for pulmonary pathogenic colonisation and infection by Pseudomonas aeruginosa in cystic fibrosis patients. Clin Microbiol Infect 2005;11:690-703.

43 Ericsson-Hollsing A, Granström M, Vasil M, et al: Prospective study of serum antibodies to Pseudomonas aeruginosa exoproteins in cystic fibrosis. J Clin Microbiol 1987;25: 1868-1874.

44 Cheer SM, Waugh J, Noble S: Inhaled tobramycin (TOBI): a review of its use in the management of Pseudomonas aeruginosa infections in patients with cystic fibrosis. Drugs 2003;63:2501-2520.

45 Blumer JL, Saiman L, Konstan MW, Melnick D: The efficacy and safety of meropenem and tobramycin vs ceftazidime and tobramycin in the treatment of acute pulmonary exacerbations in patients with cystic fibrosis. Chest 2005;128:2336-2346. 
46 Strandvik B, Hjelte L, Malmborg AS, Widén B: Home intravenous antibiotic treatment of patients with cystic fibrosis. Acta Paediatr Scand 1992;81:340-344.

-47 Parmar JS, Nasser S: Antibiotic allergy in cystic fibrosis. Thorax 2005;60:517-520.

48 Dezateux C, Walters S, Balfour-Lynn I: Inhaled corticosteroids for cystic fibrosis. The Cochrane Database Systematic Reviews 2000;2:CD001915.

49 Villa MP, Pagani J, Lucidi V, et al: Nocturnal oximetry in infants with cystic fibrosis. Arch Dis Child 2001;84:50-54.

50 Mallory GB, Fullmer JJ, Vaughan DJ: Oxygen therapy for cystic fibrosis. The Cochrane Database of Systematic Reviews 2005;4. DOI: 10.002/14651858. CD003884.pub2.
51 Dobbin CJ, Milross MA, Piper AJ, et al: Sequential use of oxygen and bi-level ventilation for respiratory failure in cystic fibrosis. J Cyst Fibros 2004;3:237-242.

52 Rosenbluth DB, Wilson K, Ferkol T, Schuster DP: Lung function decline in cystic fibrosis patients and timing for lung transplantation referral. Chest 2004;126:412-419

53 Grosse SD, Boyle CA, Botkin JR, et al: CDC: Newborn screening for cystic fibrosis: evalutation of benefits and risks and recommendations for state newborn screening programs. MMWR Recomm Rep 2004;53:136.

54 Dankert-Roelse JE, Mérelle ME: Review of outcomes of neonatal screening for cystic fibrosis versus non-screening in Europe. J Pediatr 2005;147:S15-S20.

55 Castellani C: Evidence for newborn screening for cystic fibrosis. Paediatr Respir Rev 2003;4:278-284.
56 Lannefors L, Lindgren A: Demographic transition of the Swedish cystic fibrosis community - results of modern care. Respir Med 2002;96:681-685.

57 Meyburg J, Hoffmann GF: Liver transplantation for inborn errors of metabolism. Transplantation 2005;80:S135-S137.

58 Fridell JA, Vianna R, Kwo PY, et al: Simultaneous liver and pancreas transplantation in patients with cystic fibrosis. Transplant Proc 2005;37:3567-3569.

59 Bech B, Pressler T, Iversen M, et al: Longterm outcome of lung transplantation for cystic fibrosis - Danish results. Eur J Cardiol Thorac Surg 2004;26:1180-1186.

60 Ganesh JS, Rogers CA, Bonser RS, Banner NR: Outcome of heart-lung and bilateral sequential lung transplantation for cystic fibrosis: a UK national study. Eur Respir J 2005;25:964-969. 